Теорія Ймовір. та Матем. Статист. Вип. 75, 2006
Theor. Probability and Math. Statist. No. 75, 2007, Pages 1-8 S 0094-9000(07)00709-0

Article electronically published on January 23, 2008

\title{
THE MOMENTS OF THE ARTIFICIAL REGENERATION FOR AN ASYMPTOTICALLY DEGENERATE FAMILY OF MARKOV FUNCTIONALS
}

UDC 519.21

\author{
S. V. DEGTYAR'
}

\begin{abstract}
We construct moments of the artificial regeneration satisfying certain conditions for a homogeneous ergodic Markov process related to a Markov functional in the scheme of series.
\end{abstract}

\section{INTRODUCTION}

Let $X(t)$ be a homogeneous nonperiodic ergodic Markov process [1] with either discrete or continuous time and let $X$ assume values in a phase space $(E, \mathcal{B})$. For the sake of simplicity, we assume that $E$ is a complete separable metric space, $\mathcal{B}$ is the $\sigma$-algebra of Borel sets, and the process $X(t)$ is stochastically continuous in the case of continuous time. The transition probability of the process $X(t)$ is denoted by $P(t, x, A), t \geq 0$, $x \in E, A \in \mathcal{B}$, and the invariant probability distribution is denoted by $\pi(A), A \in \mathcal{B}$.

Definition 1. A stochastic process $\xi(t)$ is called a Markov functional of a process $X(t)$ if the pair $\{X(t), \xi(t)\}$ is a homogeneous Markov process (generally speaking, $\{X(t), \xi(t)\}$ is a terminating process). We assume that the processes $X(t)$ and $\xi(t)$ are defined on a common probability space $(\Omega, \mathcal{B}, \mathrm{P})$.

In what follows we consider Markov functionals with a countable number of states $I=\{1,2, \ldots\}$. Trajectories of the process $\{X(t), \xi(t)\}$ are assumed to be right continuous and such that the left limits exist with probability one in the case of continuous time.

Denote by $\mathrm{P}_{x, i}$ the regular conditional probability given $X(0)=x$ and $\xi(0)=i$.

Consider a family of Markov functionals $\xi_{\varepsilon}(t)$ constructed from the process $X(t)$ where $\varepsilon>0$ is a small parameter. We further assume that

$$
\xi_{\varepsilon}(0)=\xi(0), \quad \varepsilon>0 .
$$

Definition 2. A family of Markov functionals $\xi_{\varepsilon}(t)$ satisfying condition (1) is called asymptotically degenerate if

$$
\lim _{\varepsilon \rightarrow 0} \mathrm{P}_{x, i}\left\{\xi_{\varepsilon}(t) \neq i\right\}=0
$$

for all $x \in E, i \in I$, and $t \geq 0$.

The latter condition is equivalent to the condition that the joint distribution of the pair $\left\{X(t), \xi_{\varepsilon}(t)\right\}$ converges to the distribution of the pair $\{X(t), \xi(0)\}$ as $\varepsilon \rightarrow 0$.

2000 Mathematics Subject Classification. Primary 60K15, 60J25.

Key words and phrases. Markov functionals, homogeneous ergodic processes. 
Since we allow the process $\xi_{\varepsilon}(t)$ to terminate, the expression $\mathrm{P}\left\{\xi_{\varepsilon}(\tau)=j\right\}$ is understood as $\mathrm{P}\left\{\xi_{\varepsilon}(\tau)=j, \tau<\zeta_{\varepsilon}\right\}$ (which is common in the theory of Markov processes) where $\zeta_{\varepsilon}$ is the lifetime of the process $\xi_{\varepsilon}(t)$.

According to the terminology accepted in the book [2], a random variable $\tau$ is called a moment of an artificial regeneration if

1) $\tau$ is a Markov moment of intervention in the evolution of the process $X(t)$ in the sense of the book [1;

2) the random variables $X(0)$ and $X(\tau)$ are independent.

It is shown in Chapter 2 of [1], that there exists a moment of artificial regeneration $\tau$ such that

$$
\mathrm{P}_{x}\{X(\tau) \in A\}=\rho(A), \quad x \in E, A \in \mathcal{B}, \quad \mathbb{P}_{\rho} \tau<\infty
$$

The measure $\rho$ above is equivalent to the measure $\pi$ (in the sense of absolute continuity). Here $\mathbb{P}_{\rho}=\int_{E} \rho(d x) \mathrm{P}_{x}$.

The construction of Markov intervention moments for Markov processes used in [1] is based on an exponential random variable being independent of the process $X(t)$ (see Chapter 2, Section 3 in [1]). Alternatively, one can use an exponential random variable increased by 1 and being independent of the process $X(t)$. This construction leads to the Markov moment of intervention such that

$$
\tau \geq 1 \quad \mathrm{P}_{x, i} \text {-a.e. }
$$

for all $x \in E$ and $i \in I$ where "a.e." stands for the "almost everywhere". The moment $\tau$ generates a sequence of Markov moments of intervention

$$
\tau_{0}=0<\tau=\tau_{1}<\tau_{2}<\cdots<\tau_{n} \rightarrow \infty
$$

such that $X\left(\tau_{1}\right), X\left(\tau_{2}\right), \ldots, X\left(\tau_{n}\right), \ldots$ is a sequence of independent identically distributed random variables with a common probability distribution $\rho$. These random variables do not depend on $X(0)$ and are such that

$$
\mathrm{P}_{x}\left\{X\left(\tau_{n}\right) \in A\right\}=\rho(A)
$$

Put $X_{n}=X\left(\tau_{n}\right)$. Let a family of $\mathcal{B} \times \mathcal{B}$ measurable functions $p_{\varepsilon}(x, y), \varepsilon>0$, be such that $0 \leq p_{\varepsilon}(x, y) \leq 1$ and $\lim _{\varepsilon \rightarrow 0} p_{\varepsilon}(x, y)=1$ for all $x, y \in E$.

An integer-valued stopping time $m$ with respect to an admissible flow

$$
\mathcal{M}_{n}, \quad n=0,1,2, \ldots
$$

(see Chapter 2, Section 1 in [1]) is called an admissible stopping time.

Put

$$
\begin{gathered}
\sigma=\tau_{m} \\
\tilde{p}_{\varepsilon}(x, y)=\mathrm{P}\left[p_{\varepsilon}\left(x, X_{1}\right) p_{\varepsilon}\left(X_{1}, X_{2}\right) \cdots p_{\varepsilon}\left(X_{m-1}, y\right)\right]=\mathrm{P}_{x}\left[\prod_{k=1}^{m} p_{\varepsilon}\left(X_{k-1}, X_{k}\right) \mid X_{m}=y\right] .
\end{gathered}
$$

The proof of the following result can be found in [1] (see Theorem 3 of Chapter 2 in [1]). 
Lemma 1. Given an arbitrary $\delta>0$, there exist a set $\widetilde{E} \in \mathcal{B}$ and an admissible stopping time $m$ such that $\pi(\widetilde{E})>1-\delta$ and

$$
\begin{gathered}
\inf _{x \in \widetilde{E}} \inf _{y \in \widetilde{E}} \widetilde{p}_{\varepsilon}(x, y) \underset{\varepsilon \rightarrow 0}{\longrightarrow} 1, \\
\mathrm{P}_{x}\{X(\sigma) \in A\}=\pi(A \widetilde{E}) / \pi \widetilde{E}, \\
\sup _{x \in \widetilde{E}} \sup _{y \in \widetilde{E}} \mathrm{P}_{x}[\sigma \mid X(\sigma)=y]<\infty, \\
\sup _{x \in \widetilde{E}} \mathrm{P}_{x}(\sigma, \sigma>n) \underset{n \rightarrow \infty}{\longrightarrow} 0, \\
\mathrm{P}_{x}\{\sigma \geq 1\}=1 .
\end{gathered}
$$

\section{MAIN RESUlts}

Our first aim is to generalize Lemma 1 to the case of matrix-valued functions. Let

$$
P_{\varepsilon}(x, y)=\left\|p_{\varepsilon}^{i j}(x, y)\right\|_{i, j=1}^{\infty}
$$

be a family of $\mathcal{B} \times \mathcal{B}$-measurable matrix-valued functions such that $p_{\varepsilon}^{i j}(x, y) \geq 0$,

$$
\sum_{j=1}^{\infty} p_{\varepsilon}^{i j}(x, y) \leq 1,
$$

and

$$
\lim _{\varepsilon \rightarrow 0} p_{\varepsilon}^{i j}(x, y)=\delta^{i j} \quad \text { for all } x, y \in E, i, j \in I .
$$

By $I=\left\|\delta^{i j}\right\|$ we denote the unit matrix and put

$$
\begin{aligned}
\widetilde{P}_{\varepsilon}(x, y) & =\left\|p_{\varepsilon}^{i j}(x, y)\right\|_{i, j=1}^{\infty}=\mathrm{P}\left[P_{\varepsilon}\left(x, X_{1}\right) P_{\varepsilon}\left(X_{1}, X_{2}\right) \cdots P_{\varepsilon}\left(X_{m-1}, y\right)\right] \\
& =\mathrm{P}_{x}\left[\prod_{k=1}^{m} P_{n}\left(X_{k-1}, X_{k}\right) \mid X_{m}=y\right] .
\end{aligned}
$$

Lemma 2. Given an arbitrary $\delta>0$, there exist a set $\widetilde{E} \in \mathcal{B}$ and an admissible stopping time $m$ such that $\pi(\widetilde{E})>1-\delta$ and

$$
\widetilde{P}_{\varepsilon}(x, y) \underset{\varepsilon \rightarrow 0}{\longrightarrow} I
$$

in the sense that

$$
\sum_{j=1}^{\infty}\left(\widetilde{p}_{\varepsilon}^{i j}(x, y)-\delta^{i j}\right) \underset{\varepsilon \rightarrow 0}{\longrightarrow} 0
$$

for all $i \in I$ and uniformly in $x, y \in \widetilde{E}$.

Proof. The inequality $\widetilde{p}_{\varepsilon}^{i i}(x, y) \geq p_{\varepsilon}^{i i}(x, y)$ and Lemma 1 imply that

$$
\widetilde{p}_{\varepsilon}^{i i}(x, y) \underset{\varepsilon \rightarrow 0}{\longrightarrow} 1
$$

uniformly in $x, y \in \widetilde{E}$. Further, $\sum_{j \neq i} \widetilde{p}_{\varepsilon}^{i j}(x, y) \leq 1-\widetilde{p}_{\varepsilon}^{i i}(x, y)$, whence

$$
\sum_{j \neq i} \widetilde{p}_{\varepsilon}^{i j}(x, y) \underset{\varepsilon \rightarrow 0}{\longrightarrow} 0
$$

uniformly in $x, y \in \widetilde{E}$. The lemma is proved. 
Now we consider the set $\widetilde{E}$ defined as in Lemma 2 and put $\widetilde{X}_{k}=X\left(\sigma_{k}\right)$ where

$$
0=\sigma_{0}<\sigma=\sigma_{1}<\sigma_{2}<\cdots<\sigma_{k}<\cdots
$$

is the sequence of moments of the Markov intervention constructed from the moment $\sigma$. Put $\widetilde{\pi}(A)=\pi(A \widetilde{E}) / \pi(\widetilde{E})$ and note that the random variables $\widetilde{X}_{0}, \widetilde{X}_{1}, \ldots, \widetilde{X}_{n}, \ldots$ are jointly independent. Moreover the random variables $\widetilde{X}_{1}, \ldots, \widetilde{X}_{n}, \ldots$ are identically distributed with the joint distribution $\pi$ and $\mathrm{P}_{\tilde{\pi}} \sigma<\infty$.

The invariant probability distribution $\pi$ of the process $X(t)$ is expressed in terms of the measures $\rho$ and $\pi$ as follows (see Theorem 3 in Chapter 2 of [1]):

$$
\int_{E} \pi(d x) f(x)=\frac{1}{\mathrm{P}_{\rho} \tau} \mathrm{P}_{\rho} \int_{0}^{\tau} f(X(s)) d s=\frac{1}{\mathrm{P}_{\pi} \sigma} \mathrm{P}_{\pi} \int_{0}^{\tau} f(X(s)) d s .
$$

Let a set $D \in \widetilde{E}$ be such that $\widetilde{\pi}(D)>0$. Then we put

$$
\begin{gathered}
\nu=\nu(D)=\nu_{1}(D)=\min \left\{k \geq 1: \widetilde{X}_{k} \in D\right\}, \\
\nu_{k}(D)=\min \left\{k>\nu_{k-1}(D): \widetilde{X}_{k} \in D\right\}, \\
\sigma_{k}(D)=\sigma_{\nu_{k}(D)} .
\end{gathered}
$$

Then

$$
\nu_{k}(\widetilde{E})=k, \quad \sigma_{k}(\widetilde{E})=\sigma_{k} .
$$

According to the definition of the set $\widetilde{E}$ we have

$$
\inf _{x \in \widetilde{E}} \inf _{y \in \widetilde{E}} \widetilde{p}_{\varepsilon}^{i i}(x, y) \underset{\varepsilon \rightarrow 0}{\longrightarrow} 1
$$

for all $i \in I$. Further we introduce the notation

$$
\begin{aligned}
Q_{\varepsilon}(x, y) & =\left\|q_{\varepsilon}^{i j}(x, y)\right\|_{i, j=1}^{\infty}=\mathrm{P}\left[\widetilde{P}_{\varepsilon}\left(x, \widetilde{X}_{1}\right) \widetilde{P}_{\varepsilon}\left(\widetilde{X}_{1}, \widetilde{X}_{2}\right) \cdots \widetilde{P}_{\varepsilon}\left(\widetilde{X}_{\nu-1}, y\right)\right] \\
& =\mathrm{P}_{x}\left[\prod_{k=1}^{\nu} \widetilde{P}_{\varepsilon}\left(\widetilde{X}_{k-1}, w t X_{k}\right) \mid \widetilde{X}_{\nu}=y\right]
\end{aligned}
$$

for a given set $D \in \widetilde{E}$.

The moment $\sigma(D)=\sigma_{\nu(D)}$ possesses the properties of the moment $\sigma$, namely

$$
\begin{gathered}
\mathrm{P}\{X(\sigma(D)) \in A\}=\pi_{D}(A), \\
\mathrm{P}_{x}\{\sigma(D) \geq 1\}=1, \\
\sup _{x \in D} \sup _{y \in D} \mathrm{P}_{x}[\sigma(D) \mid X(\sigma(D))=y]<\infty, \\
\sup _{x \in D} \mathrm{P}_{x}[\sigma(D), \sigma(D)>n] \underset{n \rightarrow \infty}{\longrightarrow} 0 .
\end{gathered}
$$

(Here and in what follows $\pi_{D}(A)=\pi(A D) / \pi(D)$ by definition.) Moreover,

$$
\int_{E} f(x) \pi(d x)=\frac{1}{\mathrm{P}_{\pi_{D}} \sigma(D)} \mathrm{P}_{\pi_{D}} \int_{0}^{\sigma(D)} f(X(s)) d s
$$

for all bounded measurable functions $f$.

It follows from (2) that

$$
\mathrm{P}_{x, i}\left\{\xi_{\varepsilon}(\tau) \neq i\right\} \underset{\varepsilon \rightarrow 0}{\longrightarrow} 0
$$

for all $x \in E$ and $i \in I$, since $\mathrm{P}_{x}\{\tau<\infty\}=1$.

Putting

$$
p_{\varepsilon}^{i j}(x, y)=\mathrm{P}_{x, i}\left\{\xi_{\varepsilon}(\tau)=j \mid X(\tau)=y\right\}
$$


and applying Lemmas 1 and 2 we prove that there exist a moment $\sigma(D)$ of the Markov intervention in the evolution of the processes $X(t),\left\{X(t), \xi_{\varepsilon}(t)\right\}$ and a set $D \in \mathcal{B}$ such that $\pi(D)>0$ and

$$
\begin{gathered}
\mathrm{P}_{x}\{X(\sigma(D)) \in A\}=\pi_{D}(A), \\
\sup _{x \in D}\left[1-\mathrm{P}_{x, i}\left\{\xi_{\varepsilon}(\sigma(D))=i\right\}\right] \underset{\varepsilon \rightarrow 0}{\longrightarrow} 0, \\
\sup _{x \in D} \mathrm{P}(\tau, \tau>T) \underset{T \rightarrow \infty}{\longrightarrow} 0 \quad \mathrm{P}_{x} \text {-a.e., } \tau \geq 1,
\end{gathered}
$$

for all $i \in I$.

By analogy with (7) one can easily get that

$$
\inf _{x \in D} \inf _{y \in D} q_{\varepsilon}^{i i}(x, y) \underset{\varepsilon \rightarrow 0}{\longrightarrow} 1
$$

for all $i \in I$.

Fix $0 \leq \alpha \leq 1$ and put

$$
\left\|f_{\varepsilon, \alpha}^{i l}(x)\right\|_{i, l=1}^{\infty}=(1-\alpha) \sum_{k=0}^{\infty} \alpha^{k} Q_{\varepsilon}^{k}(x)
$$

where

$$
\begin{gathered}
Q_{\varepsilon}^{k}(x)=\mathrm{P}_{x} \prod_{j=1}^{k} Q_{\varepsilon}\left(\widetilde{X}_{j-1}, \widetilde{X}_{j}\right), \\
\left\|g_{\varepsilon, \alpha}^{i l}(x)\right\|_{i, l=1}^{\infty}=\mathrm{P}_{x} \sum_{k=0}^{\nu-1} \alpha^{k} \prod_{j=1}^{k} Q_{\varepsilon}\left(\widetilde{X}_{j-1}, \widetilde{X}_{j}\right), \\
\left\|R_{\varepsilon, \alpha}^{i j}(x, A)\right\|_{i, j=1}^{\infty}=\mathrm{P}_{x}\left[\alpha^{\nu} \prod_{j=1}^{\nu} Q_{\varepsilon}\left(\widetilde{X}_{j-1}, \widetilde{X}_{j}\right), \widetilde{X}_{\nu} \in A\right] .
\end{gathered}
$$

Here and in what follows $\prod_{j=1}^{0}=I$ is understood as the unit matrix.

Theorem 1. Let there exist a matrix $C=\left\|c_{i j}\right\|_{i, j=1}^{\infty}$ such that

$$
\begin{gathered}
\lim _{\varepsilon \rightarrow 0} \frac{\tilde{\pi}(D)}{\varepsilon}\left[\int_{D} \int_{D} \pi_{D}(d x) \pi_{D}(d y) q_{\varepsilon}^{i i}(x, y)-1\right]=c_{i i}, \\
\lim _{\varepsilon \rightarrow 0} \tilde{\pi}(D) \sum_{j \neq i}\left[\frac{1}{\varepsilon} \int_{D} \int_{D} \pi_{D}(d x) \pi_{D}(d y) q_{\varepsilon}^{i j}(x, y)-c_{i j}\right]=0, \\
\sum_{j} c_{i j}=0, \quad \sup _{i}\left|c_{i i}\right|<\infty .
\end{gathered}
$$

Then

$$
\sum_{j}\left\{f_{\varepsilon, \alpha}^{i j}(x)-f_{i j}(t)\right\} \underset{\substack{\varepsilon \rightarrow 0 \\ \alpha \rightarrow 1 \\ \varepsilon /(1-\alpha) \rightarrow t}}{\longrightarrow} 0
$$

for all $x \in E, t \geq 0$, and $i \in I$ where $f_{i j}(t)$ is the entry $(i, j)$ of the matrix $(I-t C)^{-1}$. In other words,

$$
\left\|f_{\varepsilon, \alpha}^{i j}(x)\right\|-(I-t C)^{-1} \rightarrow 0 \quad \text { in the sense of relation }(13)
$$

as

$$
\varepsilon \rightarrow 0, \quad \alpha \rightarrow 1, \quad \varepsilon /(1-\alpha) \rightarrow t .
$$


Proof. By the full probability formula,

$$
f_{\varepsilon, \alpha}^{i l}(x)=(1-\alpha) g_{\varepsilon, \alpha}^{i l}(x)+\sum_{j} \int_{D} R_{\varepsilon, \alpha}^{i j}(x, d y) f_{\varepsilon, \alpha}^{j l}(y)
$$

or

$$
f_{\varepsilon, \alpha}^{i l}(x)=(1-\alpha) g_{\varepsilon, \alpha}^{i l}(x)+\sum_{j} R_{\varepsilon, \alpha}^{i j} f_{\varepsilon, \alpha}^{j l}(x) .
$$

Further let $U_{\varepsilon, \alpha}^{i}(x, d y)=\sum_{k=0}^{\infty}\left(R_{\varepsilon, \alpha}^{i i}\right)^{k}(x, d y)$ be the potential of the kernel $R_{\varepsilon, \alpha}^{i i}(x, d y)$ (see [1]). After some simple algebra we obtain

$$
f_{\varepsilon, \alpha}^{i l}(x)=(1-\alpha) U_{\varepsilon, \alpha}^{i} g_{\varepsilon, \alpha}^{i l}(x)+\sum_{j \neq i} U_{\varepsilon, \alpha}^{i} R_{\varepsilon, \alpha}^{i j} f_{\varepsilon, \alpha}^{j l}(x) .
$$

Denote by $\lambda_{\varepsilon, \alpha}^{i}$ the Perron root of the kernel $R_{\varepsilon, \alpha}^{i}$ and by $e_{\varepsilon, \alpha}^{i}(x)$ and $\rho_{\varepsilon, \alpha}(A)$ the eigenfunction and eigenmeasure, respectively, that correspond to the eigenvalue $\lambda_{\varepsilon, \alpha}^{i}$, that is,

$$
\begin{aligned}
& \int_{D} R_{\varepsilon, \alpha}^{i i}(x, d y) e_{\varepsilon, \alpha}^{i}(y)=\lambda_{\varepsilon, \alpha} e_{\varepsilon, \alpha}^{i}(x), \quad x \in D, \\
& \int_{D} \rho_{\varepsilon, \alpha}^{i}(d x) R_{\varepsilon, \alpha}^{i i}(x, A)=\lambda_{\varepsilon, \alpha}^{i} \rho_{\varepsilon, \alpha}^{i}(A),
\end{aligned}
$$

We assume that

$$
\rho_{\varepsilon, \alpha}^{i}(D)=1, \quad \int_{D} e_{\varepsilon, \alpha}^{i}(x) \rho_{\varepsilon, \alpha}^{i}(d x)=1 .
$$

The existence of $e_{\varepsilon, \alpha}^{i}$ and $\rho_{\varepsilon, \alpha}^{i}(A)$ follows from

$$
\sup _{x \in D} \sup _{A \in \mathcal{B}}\left|R_{\varepsilon, \alpha}^{i i}(x, A)-\pi_{D}(A)\right| \underset{\substack{\varepsilon \rightarrow 0 \\ \alpha \rightarrow 1}}{\longrightarrow} 0
$$

and from the theorem on the perturbation of isolated parts of the spectrum [3, Chapter 4, Section 4, Theorem 3.16]. Moreover,

$$
\begin{gathered}
\sup _{x \in D}\left|e_{\varepsilon, \alpha}^{i}-1\right| \underset{\substack{\varepsilon \rightarrow 0 \\
\alpha \rightarrow 1}}{\longrightarrow} 0, \\
\sup _{A \in \mathcal{B}}\left|\rho_{\varepsilon, \alpha}^{i}(A)-\pi_{D}(A)\right| \underset{\substack{\varepsilon \rightarrow 0 \\
\alpha \rightarrow 1}}{\longrightarrow} 0 .
\end{gathered}
$$

The same theorem on the perturbation of the isolated parts of the spectrum and relation (16) imply that

$$
U_{\varepsilon, \alpha}^{i}(x, A)-\frac{1}{1-\lambda_{\varepsilon, \alpha}^{i}} e_{\varepsilon, \alpha}^{i}(x) \rho_{\varepsilon, \alpha}^{i}(A) \underset{\substack{\varepsilon \rightarrow 0 \\ \alpha \rightarrow 1}}{\longrightarrow} I(x, A)-\pi_{D}(A)
$$

uniformly in $x \in D$ and $A \in \mathcal{B}$ where

$$
I(x, A)= \begin{cases}1, & x \in A, \\ 0, & x \notin A,\end{cases}
$$

is the unit kernel. Using relations (16)-(18) and the obvious inequality $R_{\varepsilon, \alpha}^{i i} \leq \pi_{D}(A)$ one can easily prove that

$$
1-\lambda_{\varepsilon, \alpha}^{i} \sim\left(1-\int_{D} \int_{D} \pi_{D}(d x) \pi_{D}(d y) q_{\varepsilon}^{i i}(x, y)+\frac{(1-\alpha)}{\widetilde{\pi}(D)}\right)
$$

as $\varepsilon \rightarrow 0$ and $\alpha \rightarrow 1$. The latter relation together with assumption (10) implies that if $\varepsilon$ and $\alpha$ vary as stated in (14), then

$$
\frac{1-\lambda_{\varepsilon, \alpha}^{i}}{1-\alpha} \underset{(14)}{\longrightarrow} \frac{1-t c_{i i}}{\tilde{\pi}(D)} .
$$


Now we obtain from (10) and (17)-(21) that

$$
(1-\alpha) U_{\varepsilon, \alpha}^{i}(x, A) \underset{(14)}{\longrightarrow} \frac{\tilde{\pi}(D)}{1-t c_{i i}} \cdot \pi_{D}(A)
$$

uniformly in $x \in D$ and $A \in \mathcal{B}$.

It is also clear that relation (9) implies that

$$
g_{\varepsilon, \alpha}^{i l}(x) \underset{\substack{\varepsilon \rightarrow 0 \\ \alpha \rightarrow 1}}{\longrightarrow} \delta_{i l} \mathrm{P}_{x} \nu=\delta_{i l} / \widetilde{\pi}(D)
$$

uniformly in $x \in D$. Thus

$$
(1-\alpha) U_{\varepsilon, \alpha}^{i} g_{\varepsilon, \alpha}^{i}(x) \underset{(14)}{\longrightarrow} \frac{\delta_{i l}}{1-t c_{i i}}
$$

uniformly in $x \in D$.

Since

$$
\sum_{j \neq i} R_{\varepsilon, \alpha}^{i j}(x, A) \underset{\substack{\varepsilon \rightarrow 0 \\ \alpha \rightarrow 1}}{\longrightarrow} 0
$$

uniformly in $x \in D$ and $A \in \mathcal{B}$, we get from (19) that

$$
\sum_{j \neq i}\left[U_{\varepsilon, \alpha}^{i} R_{\varepsilon, \alpha}^{i j}(x, A)-e_{\varepsilon, \alpha}^{i} \rho_{\varepsilon, \alpha}^{i j}(A)\right] \underset{\varepsilon \underset{\alpha \uparrow 1}{\longrightarrow} 0}{\longrightarrow} 0
$$

uniformly in $x \in D$ and $A \in \mathcal{B}$ where

$$
\rho_{\varepsilon, \alpha}^{i j}(A)=\frac{1}{1-\lambda_{\varepsilon, \alpha}} \int_{D} \rho_{\varepsilon, \alpha}^{i}(d x) R_{\varepsilon, \alpha}^{i j}(x, A) .
$$

Note that $\rho_{\varepsilon, \alpha}^{i j}(A) \geq 0$ and

$$
\sum_{j \neq i} \rho_{\varepsilon, \alpha}^{i j}(D) \leq \frac{1}{1-\lambda_{\varepsilon, \alpha}^{i}} \int_{D} \rho_{\varepsilon, \alpha}^{i}(d x)\left[1-R_{\varepsilon, \alpha}^{i i}(x, D)\right]=1 .
$$

This together with relation (17) yields

$$
\sum_{j \neq i}\left[U_{\varepsilon, \alpha}^{i} R_{\varepsilon, \alpha}^{i j}(x, A)-\rho_{\varepsilon, \alpha}^{i j}(A)\right] \underset{(14)}{\longrightarrow} 0
$$

uniformly in $x \in D$ and $A \in \mathcal{B}$. Since $f_{\varepsilon, \alpha}^{i l}(x)$ is uniformly bounded, we obtain

$$
\sum_{l=1}^{\infty}\left\{f_{\varepsilon, \alpha}^{i l}(x)-\left[\frac{\delta_{i l}}{1-t c_{i i}}+\frac{t}{1-t c_{i i}} \sum_{j \neq i} \rho_{\varepsilon, \alpha}^{i j}(d y) f_{\varepsilon, \alpha}^{j l}(y)\right]\right\} \underset{(14)}{\longrightarrow} 0
$$

uniformly in $x \in D$. Denote the expression in the square brackets by $\widehat{f}_{\varepsilon, \alpha}^{i l}$. Then

$$
\sum_{l=1}^{\infty}\left[f_{\varepsilon, \alpha}^{i l}(x)-\widehat{f}_{\varepsilon, \alpha}^{i l}\right] \underset{(14)}{\longrightarrow} 0
$$

whence

$$
\sum_{l=1}^{\infty}\left\{\widehat{f}_{\varepsilon, \alpha}^{l l}(x)-\left[\frac{\delta_{i l}}{1-t c_{i i}}+\frac{t}{1-t c_{i i}} \sum_{j \neq i} c_{i j} \widehat{f}_{\varepsilon, \alpha}^{j l}\right]\right\} \underset{(14)}{\longrightarrow} 0 .
$$

Therefore we derive from (22) and (23) that (13) holds uniformly in $x \in D$. 


\section{Concluding Remarks}

The main ideas for constructing the so-called moments of regeneration are due to Kovalenko [4, Nummelin [5], and Athreya, McDonald, and Ney [6]. The existence of the moments of regeneration allows one to apply powerful analytical tools of renewal theory to the investigation of the limit behavior of transition probabilities. Combining the asymptotic results concerning solutions of the Markov renewal equation with the methods of artificial moments of regeneration we are able to investigate the behavior of Markov functionals with an infinite number of states.

\section{BIBLIOGRAPHY}

1. V. M. Shurenkov, Ergodic Theorems and Related Problems, Moscow, "Nauka", 1989; English transl., VSP International Science Publishers, Leiden, 1998. MR1690361 (2000i:60002)

2. N. I. Portenko, A. V. Skorokhod, and V. M. Shurenkov, Markov processes, Itogi Nauki i Tekhniki, Current problems in mathematics. Fundamental directions, vol. 46, Vsesoyuz. Inst. Nauchn. i Tekhn. Inform., Moscow, 1989, pp. 5-245. (Russian) MR.1039619 (91j:60113b)

3. T. Kato, Perturbation Theory for Linear Operators, Springer-Verlag, Berlin-New York, 1966. MR0203473 (34:3324)

4. I. N. Kovalenko, Limit theorems of reliability theory, Kibernetika 6 (1977), 106-116. (Russian) MR0518465 (58:24589)

5. E. Nummelin, Uniform and Ratio Limit Theorems for Markov Renewal and Semi-Regenerative Processes on a General State Space, Report Helsinki Univ. Technol. Inst. Math. Espoo, vol. A 98, 1977. MR507729 (80a:60114)

6. K. B. Athreya, D. McDonald, and P. Ney, Limit theorems for semi-Markov processes and renewal theory for Markov chains, Ann. Probab. (1978), no. 6, 788-797. MR.503952 (80f:60076)

Department of Higher Mathematics, Vadym Hetman Kyiv National Economic University, Peremogy Avenue 54/1, Kyiv 03057, Ukraine

Received 6/SEP/2005

Translated by N. SEMENOV 